\title{
A Novel Hydrodynamic Injection Mouse Model of HBV Genotype C for the Study of HBV Biology and the Anti-Viral Activity of Lamivudine
}

\author{
Xiumei Li, ${ }^{1}$ Guangze Liu, ${ }^{1}$ Meijuan Chen, ${ }^{1}$ Yang Yang, ${ }^{1}$ Yong Xie, and Xiangping Kong ${ }^{1,{ }^{*}}$ \\ ${ }^{1}$ Liver Disease Key Lab, 458 Hospital of PLA, Guangzhou, China \\ ${ }^{*}$ Corresponding Author: Xiangping Kong, Liver Disease Key Lab, 458 Hospital of PLA, 801 Dongfengdong Road, 510600, Guangzhou, Guangdong, China. Tel: +86-2087395343, Fax: \\ +86-2087371180, E-mail: xiangping_kong1@hotmail.com
}

Received 2015 November 12; Revised 2016 January 6; Accepted 2016 January 15.

\begin{abstract}
Background: Absence of an immunocompetent mouse model of persistent hepatitis B virus (HBV) infection has hindered the research of HBV infection and the development of antiviral medications.

Objectives: In the present study, we aimed to develop a novel HBV genotype $\mathrm{C}$ mouse model by hydrodynamic injection (HI) and then used it to evaluate the antiviral activity of lamivudine.

Materials and Methods: A quantity of $15 \mu \mathrm{g}$ of HBV plasmid [pcDNA3.1 (+)-HBV1.3C], adeno-associated virus-HBV1.3C (pAAV-HBV1.3C) or pAAV-HBV1.2A) were injected into male C57BL/6 mice, by HI, accounting for a total of 13 mice per group. Then, lamivudine was administered to mice with sustained HBV viremia, for 4 weeks. Real-time polymerase chain reaction (RT-PCR), enzyme-linked immunosorbent assay (ELISA) and immunohistochemistry methods were used to detect HBsAg, HBeAg, HBsAb, HBcAg and HBV DNA, in serum or liver of the mice, at indicated time points.

Results: In 60\% of the mice injected with pcDNA3.1 (+)-HBV1.3C, HBsAg, HBeAg, HBcAg and HBV DNA persisted for $>20$ weeks in liver, post-injection, with no HBsAb appearance. Meanwhile, no significant inflammation was observed in these mice. Compared with pAAVHBV1.2A and pAAV-HBV1.3C, pcDNA3.1(+)-HBV1.3C administration led to higher and longer HBV viremia. Furthermore, serum HBV DNA was significantly reduced by lamivudine, after 4 weeks administration, and returned to the original level, after ceasing administration for 1 week, in the mice.

Conclusions: In conclusion, our observations indicated that pcDNA3.1(+)-HBV1.3C was superior to AAV/HBV plasmid for establishment of persistent $\mathrm{HBV}$ infection by $\mathrm{HI}$, in vivo, and this mouse model could be useful for studies of hepatitis virology and for the development of innovatory treatments for $\mathrm{HBV}$ infections.
\end{abstract}

Keywords: Hepatitis B virus, Intravenous, Injections, Models, Animal, Lamivudine

\section{Background}

It is considered that around 400 million people worldwide suffer from chronic HBV infection, which results in chronic liver infection and potentially death, because of liver failure and hepatocellular carcinoma (1). The hepatitis B virus (HBV) infection is still a serious public health problem, all over the world.

To date, HBV has been differentiated into $10 \mathrm{HBV}$ genotypes, according to genome sequence (2). Multiple studies have reported that different genotypes show different geographical distribution and are related to disease progression, response to antiviral treatment and prognosis (3). In Asia, the dominant genotypes of HBV are genotypes B and $C$ (4). To address this issue, it is important to develop HBV mouse models with the characterized HBV strains.

To study the virology and pathogenesis of HBV infection, several animal models have been established to mimic hepatic HBV infection and the immune response against HBV (5-7). However, because HBV infection shows strict host specificity there are still significant limitations, due to the lack of immunocompetent animal models $(8,9)$. As a result, HBV-related research has been greatly hampered.
Several physical methods were developed for gene delivery in vivo, among which hydrodynamic injection (HI), which represents a rapid injection of a large volume of DNA through the tail vein, was the simplest and highly effective method $(10,11)$. The HI was used widely in multiple researches, such as gene therapy studies, gene function analyses, and establishment of animal models (12-14).

It was reported that a single injection of naked plasmid DNA could transfect approximately $40 \%$ of mice hepatocytes and the related protein could be expressed for up to 9 months $(15,16)$. Nowadays, mice transfected by HI are useful tools for studying the host immune response, the pathophysiology of HBV-related disease and several antiviral drugs, as well (17-19). Although the HBV HI mouse models of HBV genotype A and B were already established $(20,21)$, HBV genotype C, which is dominant in Asia, especially in China, was not.

\section{Objectives}

The present study aimed to establish a HBV genotype C

Copyright (C) 2016, Kowsar Corp. This is an open-access article distributed under the terms of the Creative Commons Attribution-NonCommercial 4.0 International License (http://creativecommons.org/licenses/by-nc/4.0/) which permits copy and redistribute the material just in noncommercial usages, provided the original work is properly cited. 
HI mouse model with sustained HBV viremia and to demonstrate whether this mouse model could be used as a useful tool for the study of HBV infection and evaluation of antiviral medications.

\section{Materials and Methods}

\subsection{Study Animals}

Six to eight weeks old C57BL/6 male mice (from the breeding colonies of the experimental animal center, Guangdong province, China) were kept under specificpathogen-free (SPF) conditions, in the clean rooms, at the animal center for medical experiment, liver disease key lab, 458 hospital of PLA, Guangzhou, China. These experiments were approved by the ethics review committee for animal experimentation of 458 hospital of PLA, Guangzhou, China, and were conducted according to the NIH guide for the care and use of laboratory animals (22).

\subsection{Construction of Replication Competent HBV Genotype C Clones}

The plasmid pAAV-HBV1.2A, which contains a 1.2 fold over-length HBV genotype A genome (nt 1400-3182-1987), was offered by Professor Huang etal. (20) (graduate institute of clinical medicine, college of medicine, national Taiwan university, Taipei, Taiwan). The plasmid pcDNA3.1 (+)-HBV1.3C, containing a 1.3 fold genotype C HBV genome (nt 1387-3215-2598, GenBank: EU560440.1) was provided by ProfessorXu (institute of infectious diseases and liver failure research center, 302 hospital of PLA, Beijing, China). Then, we cloned the 1.3 fold genotype $C$ HBV genome into the vector PAAV-mCMV-IRES-ZsGreen (Biowit technologies, Shenzhen, China) and designated the resulting plasmid as pAAV-HBV1.3C.

\subsection{Hydrodynamic Injection Experiments}

Six to eight weeks old C57BL/6 male mice were subjected to HI, with pcDNA3.1 (+)-HBV1.3C, pAAV-HBV1.3C or pAAVHBV1.2A, with 13 mice per group. The HI was carried out as described previously (21). Briefly, $15 \mu \mathrm{g}$ of different HBV plasmid DNA was injected into the tail veins of mice, in a volume of saline, which was equivalent to $8 \%$ of the mouse body weight. The total volume was delivered within 5 - 8 seconds. The control mice received only saline.

\subsection{Serological and Biochemical Analysis}

Serum was harvested by retro-orbital bleeding. Serum alanine aminotransferase (ALT) levels were analyzed by standard spectrophotomeric methods, using commercial test reagents (Jiancheng Biotechnology, Nanjing, China).

\subsection{Detection of HBV Immunological Markers in Serum by Enzyme-Linked Immunosorbent Assay}

Mouse sera were collected on the 1 day, 7 day, 2 week, 3 - 10 week, etc. after HI. Serum HBsAg, HBeAg, HBsAb were measured by using commercial ELISA kits (Kehua Bio- engineering Co. Ltd, Shanghai, China) according to the manufacturer's instructions.

\subsection{Quantitation of HBV DNA in Serum by Real- Time Polymerase Chain Reaction}

A volume of $60 \mu \mathrm{L}$ of mouse serum was used to extract HBV DNA, following the manufacturer's instructions (Qiagen, Hilden, Germany). Then, quantitation of HBV DNA was performed by SYBR Green real-time polymerase chain reaction (RT-PCR) master mix (commercially available assay kit, TOYOBO, Osaka, Japan).

\subsection{Immunohistochemistry}

The liver tissue taken from the HBsAg-positive mice at 3 weeks after $\mathrm{HI}$ ( $3 \mathrm{wpi}$ ) and at 20 wpi with pcDNA3.1 (+)-HBV1.3C, pAAV-HBV1.3C or pAAV-HBV1.2A were fixed with $10 \%$ formalin and embedded in paraffin. The liver sections were incubated with primary antibodies (polyclonal rabbit anti-HBcAg, DAKO, Glostrup, Denmark) and the DAKO envision system. hematoxylin and eosin (HE) staining were also performed.

\subsection{Treatment With Antiviral Agents}

Six weeks after HI with pcDNA3.1 (+)-HBV1.3C, mice with stable viremia were selected and treated with antiviral medication. Lamivudine (Roche, Penzberg, Germany) was diluted in saline and a gavage was administered for 4 weeks, at a dose of $100 \mathrm{mg} / \mathrm{kg} / \mathrm{d}$.

\subsection{Statistical Analyses}

Statistical analyses were performed with the SPSS software version 16.0 (SPSS Inc, Chicago, IL, USA). Differences between the two groups or among multiple groups were analyzed by the two-tailed unpaired Student's t test and one-way ANOVA with Turkey's multiple. The results were expressed as mean \pm SD. The statistical significance of the data was considered at $\mathrm{P}<0.05$.

\section{Results}

\subsection{The Influence of Plasmid Backbone on HBV Persistence}

In the present study, the 1.3 fold HBV genotype $C$ genome was cloned into the pAAV vector to generate pAAVHBV1.3C. Then, the three plasmids (pcDNA3.1 (+)-HBV1.3C, pAAV-HBV1.3C or pAAV-HBV1.2A) were injected into C57BL/6 mice with HI, respectively, to examine whether plasmid carrying HBV genotype $C$ was able to establish persistent replication in vivo and to compare influence of plasmid backbone on HBV persistence.

Mouse serum samples were collected at the indicated time points for ELISA assay of HBsAg, HBsAb and HBeAg. As shown in Figure 1, in all three groups, the HBsAg level increased on the first day. Afterwards, the HBsAg level dropped quickly, with a concomitant fast increment of HBsAb in mice in- 
jected with pAAV-HBV1.3C, among which only $8 \%$ of the mice were positive at $3 \mathrm{wpi}$ and all mice became negative at $8 \mathrm{wpi}$. The HBsAg level, in the other two groups, declined slowly with time. Although 50\% of the mice injected with pAAVHBV1.2A were HBsAg positive for 10 weeks, all mice became negative at 12 wpi. Meanwhile, the HBsAb levels became highest among the three groups, after 8 weeks. In contrast to the other two groups, HBsAb level was much lower in mice receiving pcDNA3.1 (+)-HBV1.3C. Only $60 \%$ of the mice HI with pcDNA3.1 (+)-HBV1.3C maintained stable and high HBsAg level, with no HBsAb appearance until 20 wpi.

Additionally, HBeAg was also detected in the sera of infected mice (Figure 1 E). Only pcDNA3.1 (+)-HBV1.3C transfected mice were HBeAg-positive, indicating active HBV replication, in this mouse model.

Therefore, in contrast to pAAV-HBV1.3C and pAAV-HBV1.2A, administration of pcDNA3.1 (+)-HBV1.3C lead to longer and higher levels of HBsAg and HBeAg in the sera of injected mice and was associated with persistent viremia.

\subsection{Expression of HBCAg in Mouse Liver}

The expression of HBcAg in mouse liver was analyzed by immunohistochemistry at 3 wpi and 20 wpi. As shown in Figure 2, although HBcAg was localized to the cytoplasm and the nuclei of hepatocytes, it had the most abundance in nuclei. The HBcAg was found in the liver of HI HBsAg-positive mice, with each of the three plasmids at 3wpi. The expression of HBcAg was highest in HI mice with pAAV-HBV1.2A (Figure $2 \mathrm{~A}$ ), moderate in mice which received pcDNA3.1 (+)-HBV1.3C (Figure $2 \mathrm{~B}$ ) and lowest in those HI with pAAV-HBV1.3C (Figure $2 \mathrm{C}$ ), with $25 \%, 15 \%$ and $2 \%$ of hepatocytes expressing $\mathrm{HBcAg}$, respectively. At 20 wpi, only HI mice with pcDNA3.1 (+)-HBV1.3C were HBsAg-positive, in serum. Accordingly, 5\% HBcAg-positive

Figure 1. Persistence of HBV Markers (HBsAg, HBeAg, HBsAb and HBV DNA) After HI in Sera of Infected Mice

$\overline{\mathbf{A}}$

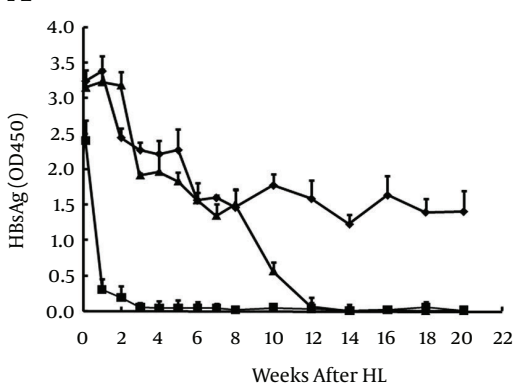

C

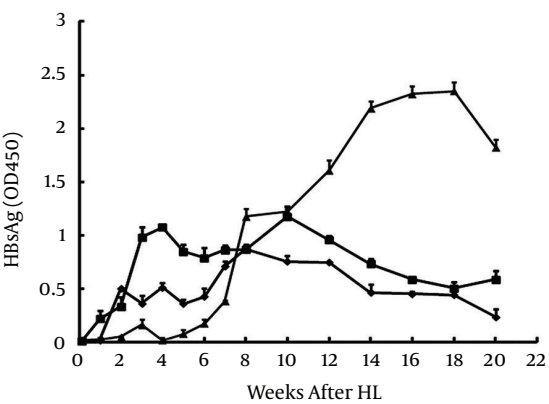

E

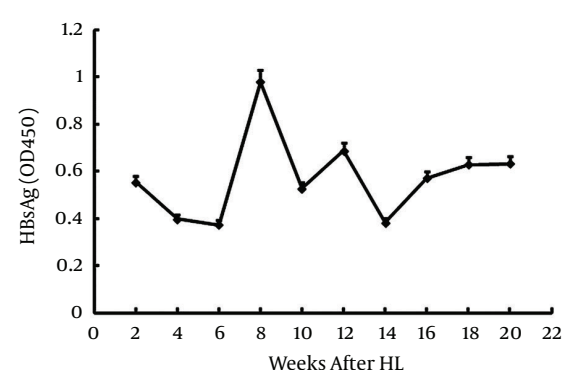

B

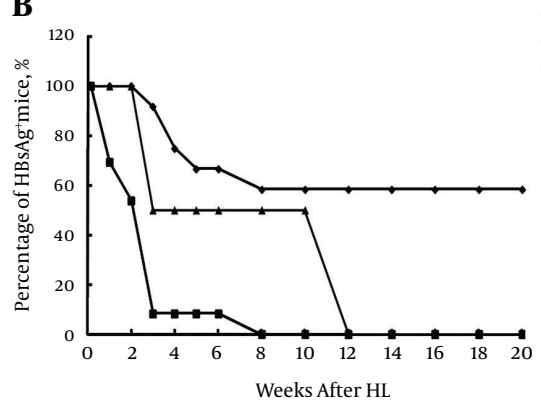

$\mathbf{D}$

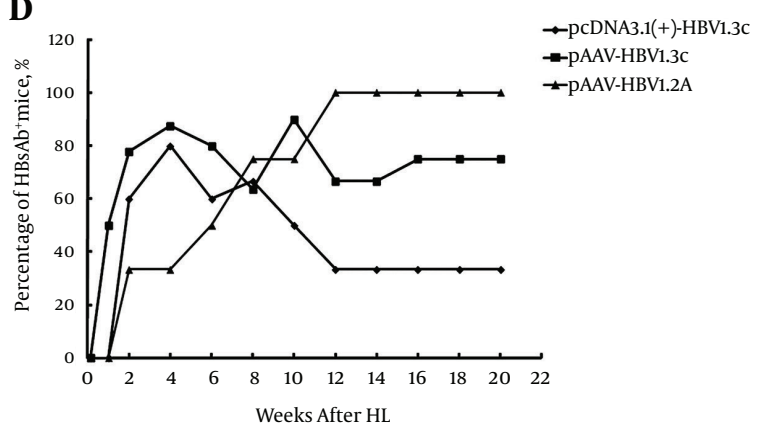

F

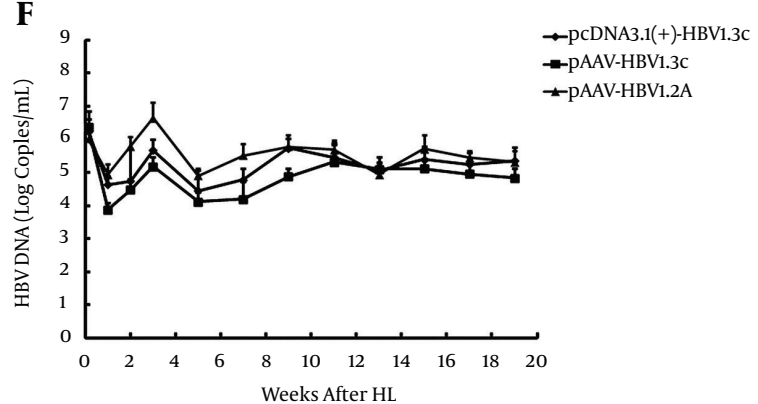

A volume of $15 \mu \mathrm{g}$ of HBV plasmid DNA (pcDNA3.1 (+)-HBV1.3C, pAAV-HBV1.3C or pAAV-HBV1.2A) was injected into the tail veins of male C57BL/6 mice. Each group included 13 mice. At indicated time points, HBsAg, HBeAg and HBsAb in sera were measured by enzyme-linked immunosorbent assay. The HBV DNA in sera was detected by real-time polymerase chain reaction (A, C, D); A, Titer of sera HBsAg; C, HBsAb; E, HBeAg (OD value) in mice after hydrodynamic injection (HI); B, The positive rate of HBsAg; D, or HBsAb; F, in mice after HI. Sera HBV DNA in mice, after HI, and expressed as the lg copies/mL. The results are shown as mean \pm SD. 
cells were still present in the liver of these mice (Figure 2 D), while the expression of HBcAg was reduced to less than $1 \%$, in pAAV-HBV1.2A DNA injection mice (Figure $2 \mathrm{E}$ ), and declined to undetectable levels in the pAAV-HBV1.3C-treated mice (Figure $2 \mathrm{~F}$ ). Even more, no HBcAg expression could be detected in HBsAg-negative mice at w3 (Figure $2 \mathrm{G}$ ) and w20 (Figure $2 \mathrm{H}$ ). Consequently, HI with pcDNA3.1 (+)-HBV1.3C lead to longer expression of HBcAg in mouse liver, compared with HI with pAAV-HBV1.3C and pAAV-HBV1.2A. The HBcAg was absent in HBsAg-negative mice.

Figure 2. Expression of HBcAg in the Liver of Mice, After Hydrodynamic Injection
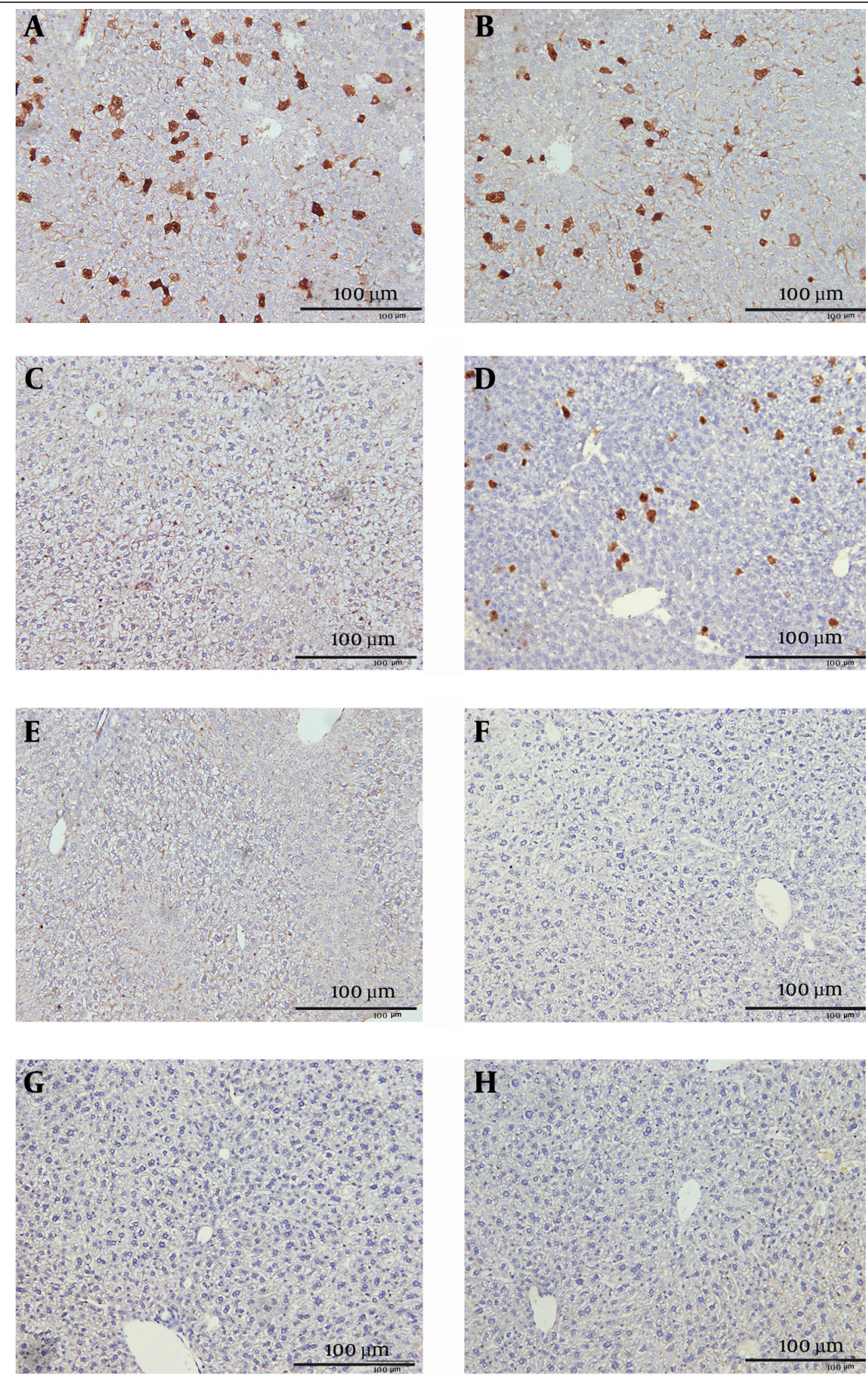

A, Immunohistochemical staining of the liver sections for HBcAg, in hepatocytes of HBsAg-positive mice, at 3 weeks (3w) after hydrodynamic injection (HI) with pAAV-HBV1.2A; B, pcDNA3.1 (+)-HBV1.3C; C, or pAAV-HBV1.3C; D, and at 20w after HI with pcDNA3.1 (+)-HBV1.3C; E, pAAV-HBV1.2A;F, pcDNA3.1 (+)-HBV1.3C; G, The HBsAg-negative mice at 3w after HI (3wpi); H, The HBsAg-negative mice at 20wpi (Original magnification:200X). Results for one representative mouse are shown. 


\subsection{Detection of HBV DNA in Serum}

The serum HBV DNA levels in HI mice were also detected at the indicated time points by RT-PCR. Figure $1 \mathrm{~F}$ showed that the HBV DNA in serum reached a peak at 3wpi and decreased thereafter in all three groups. Nevertheless, it was detectable at later time points, until w19, and kept high in the serum of HBsAg-positive mice. Mice injected with pAAV-HBV1.2A resulted in higher HBV DNA level compared with those HI with pcDNA3.1 (+)-HBV1.3C and pAAVHBV1.3C, highly correlating with the expression pattern of HBcAg in mice liver.

\subsection{Histological Analysis of Liver Sections and Transaminase Activities in Serum}

To analyze whether HBV chronic infection causes liver damage, serum samples were collected from mice infected with different plasmid and measured the levels of serum ALT. No significant increase in ALT was observed in infected mice (Figure $3 \mathrm{~A}$ ). The HE staining did not show obvious infiltrating lymphocytes or other abnormalities in the infected liver tissue (Figure $3 \mathrm{~B}$ ). These results indicated that HBV infection through $\mathrm{HI}$ did not induce detectable liver injury.
A

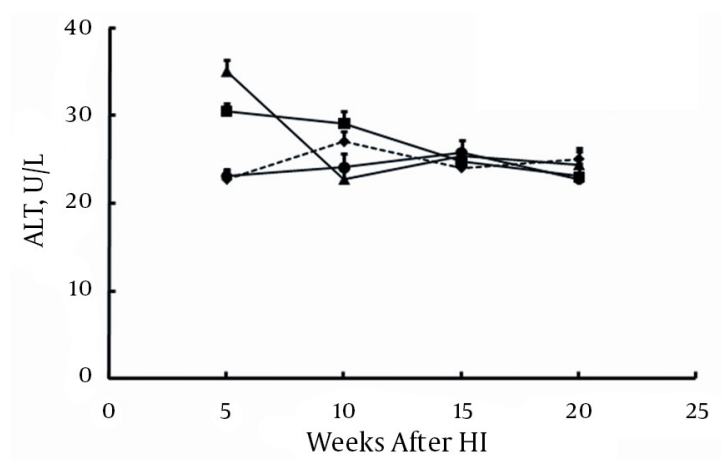

B

$3 \mathrm{~W}$
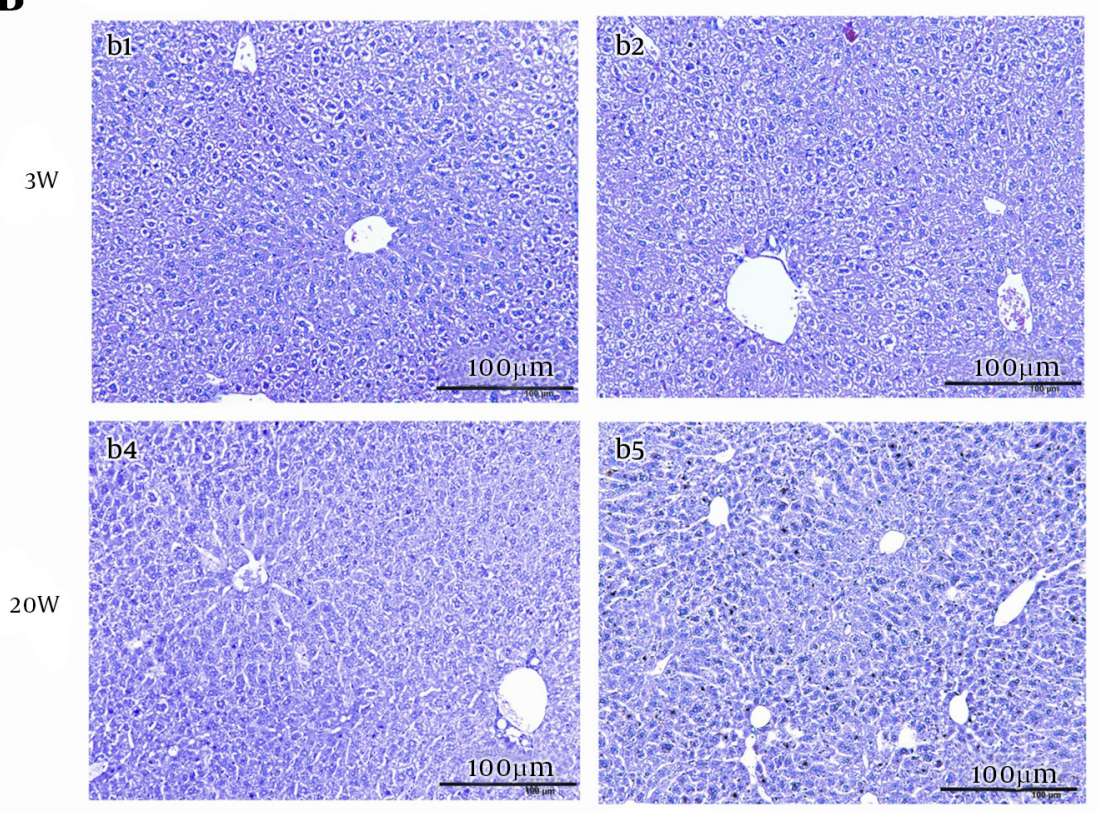

PAAV-HBV1.2A

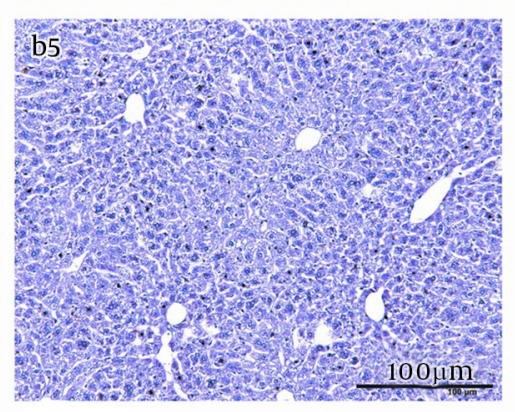

pcDNA3.1(+)-HBV1.3C

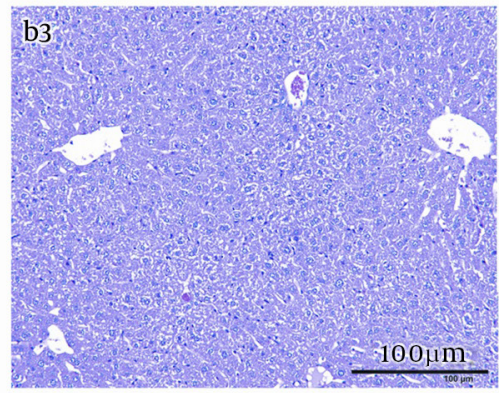

$\rightarrow-$ pcDNA3.1(+)-HBV1.3

$\rightarrow$ PAAV-HBV1.3C

$\rightarrow-$ Control

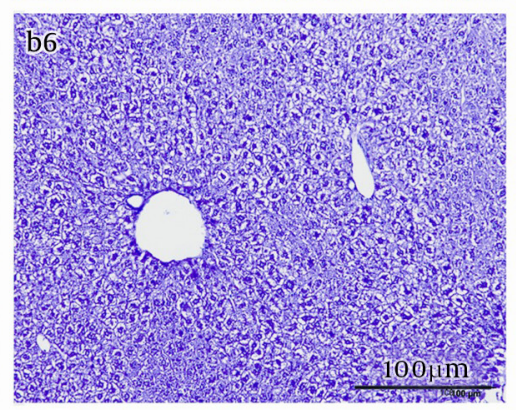

pAAV-HBV1.3c

A, Mean alanine transaminase activities in sera from mice injected with three different plasmids or 0.9\% NaCl, expressed as U/L. (B1- B6) Hematoxylin and eosin staining of liver sections from mice at 3 weeks or 20 weeks after injection with three different plasmids. (Original magnification: $200 \mathrm{X}$ ). Results for one representative mouse are shown. 
4.5. The Effect of Lamivudine on Hepatitis VirusInfected Mice

To investigate whether the mouse model established in this study can be used for the study of anti-HBV drug, six mice selected from pcDNA3.1 (+)-HBV1.3C injection group, with high and stable HBsAg level, were used to evaluate the effect of lamivudine on HBV replication. Lamivudine was diluted in saline and a gavage was administered for 4 weeks, at a dose of $100 \mathrm{mg} / \mathrm{kg} / \mathrm{d}$, starting from 6 weeks post-DNA administration. The HBV DNA levels were measured in mice sera using RT-PCR. The results showed that lamivudine significantly decreased serum HBV DNA content, after one week of administration, and this inhibitory effect lasted up to 4 weeks. The HBV DNA increased to the former level when lamivudine administration was stopped for one week (Figure 4). In control mice, no significant alteration in serum HBV DNA content was observed.

Figure 4. The Effect of Treatment With Lamivudine on HBV DNA in Sera of HBsAg-Positive Mice Hydrodynamically Injected With pcDNA3.1 (+)-HBV1.3C

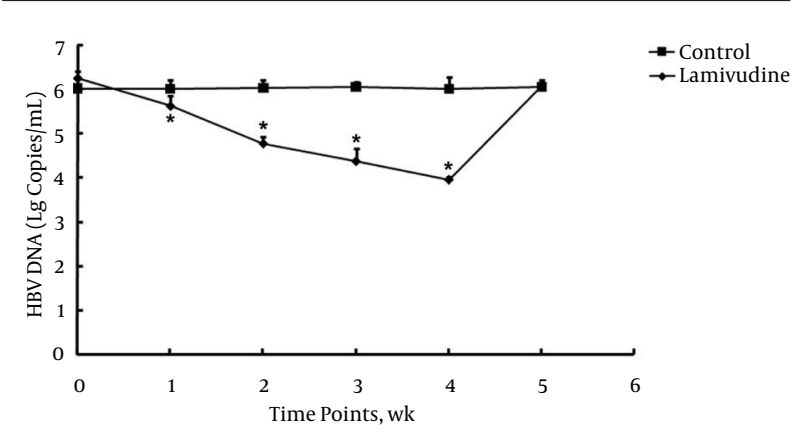

Lamivudine or saline were administered orally, every day, at a dose of 100 $\mathrm{mg} / \mathrm{kg} / \mathrm{d}$ for 4 weeks, starting from 42 days post-DNA administration. Each group included three mice. The HBV-DNA titer ( $\mathrm{g}$ copies/mL) was determined by real-time polymerase chain reaction detection, at indicated time points; $\mathrm{P}<0.05$.

\section{Discussion}

Clinical outcomes of chronic HBV infection are diverse. Among different factors, HBV genotypes may have different virological characteristics that are associated with clinical progression of infection and antiviral therapy $(3,23)$. To date, $10 \mathrm{HBV}$ genotypes (from A to J) have been identified. As HBV genotype $\mathrm{C}$ is dominant in Asia, especially in China (4), and HBV genotypes may have different virological characteristics that are associated with clinical progression of infection and antiviral therapy $(2,23$, $24)$, it is urgently needed to generate HBV mouse models with characterized HBV strains. Previously, Huang et al. and Li et al. established HBV HI mouse models of HBV genotype $A$ and $B$, respectively, and showed that a persistent replication HBV mouse model could be prepared by $\mathrm{HI}$ and plasmid backbone had influence on HBV persistence $(20,21)$.
As HBV genotype $\mathrm{C}$ is dominant in Asia, especially in China (4), we herein established a HBV genotype C HI mouse model. After HI with three different plasmids, including pcDNA3.1 (+)-HBV1.3C, pAAV-HBV1.3C and pAAV-HBV1.2A, into male C57BL/6 mice, HBsAg, HBsAb, HBeAg and HBV DNA were detected in peripheral blood, at the indicated time points. The HBcAg were detected in the liver tissue by immunohistochemical staining. Our results showed that administration of pcDNA3.1 (+)-HBV1.3C resulted in $\mathrm{HBV}$ persistence in immunocompetent $\mathrm{C} 57 \mathrm{BL} / 6$ mice. The HBsAg, HBeAg, HBV DNA in serum could be detected until 20 w in $60 \%$ of HI mice, without developing HBsAb. Immunohistochemical staining of the liver tissue for $\mathrm{HBcAg}$, at 3wpi and 20wpi, revealed that HI with pcDNA3.1 (+)-HBV1.3C lead to longer expression of HBcAg in mouse liver, compared with $\mathrm{HI}$ with the other two plasmids (pAAV-HBV1.3C and pAAV-HBV1.2A). All mice became HBsAg-negative with high HBsAb level at 8wpi, in pAAVHBV1.3C group, and at $12 \mathrm{wpi}$, in pAAV-HBV1.2A group. Although several researches reported that mice models with sustained HBV viremia after infection with a recombinant adeno-associated virus, carried a replicable HBV genome (AAV/HBV) or related plasmid $(20,25)$, others found that AAV/HBV transfer resulted in acute HBV infection (12). Our results were similar to the Chuai et al. study (12). The present results demonstrated that plasmids carrying different HBV genomes might have different ability to establish the persistent replication in mice and plasmid backbone had effect on the HBV persistence, certainly. Moreover, pcDNA3.1(+)-HBV1.3C was superior to $\mathrm{AAV} / \mathrm{HBV}$ plasmid for the development of persistent HBV infection via HI, in vivo. Nevertheless, the details still require further clarification.

Herein, we also evidenced the expression of HBcAg, which meant that there was HBV replication in the HI mice, was in agreement with the quantity of HBV DNA in mice serum. However, in view of persistent high levels of HBsAg, with less HBsAb in pcDNA3.1 (+)-HBV1.3C injection mice, while the other two groups showed reduced HBsAg level and increased HBsAb level along with the time changes, the results suggested that HBV replication was not strictly correlated with HBsAg production, as known for patients (26). These results were consistent with the previous studies $(27,28)$. Combined with the results of normal serum ALT and without significant inflammation in the liver, the present study indicated that pcDNA3.1 (+)-HBV1.3C might cause weaker anti-HBV immune response and this mouse model prepared by HI with pcDNA3.1 (+)-HBV1.3C plasmid might recapitulate viral and histological characteristics of human chronic HBV infection in the immune tolerance phase of the disease (29).

Several studies showed that mice transfected by HI were appropriate for testing antiviral therapy methods (17, $21,30)$. In this study, persistent high levels of HBV DNA, HBsAg and HBeAg in the sera of pcDNA3.1 (+)-HBV1.3C injected mice, made this model suitable for the evaluation of the efficacy of antiviral drugs. Therefore, we further in- 
vestigated the antiviral effect of lamivudine in this model. The results showed that administration of lamivudine for 4 weeks significantly reduced serum HBV DNA content. However, after stopping administration for 1 week, HBV DNA returned to the original level. Similar results were obtained following administration of lamivudine to hepatitis B patients (31), suggesting that this mouse model could mimic HBV infection in people and might be an appropriate animal model to evaluate the effect of antiviral agents.

In summary, we established an immunocompetent mouse model of HBV genotype $\mathrm{C}$ by HI. This model is valid for the study of hepatitis virology, evaluation of antiviral drugs and immunotherapies. To our knowledge, for the first time, we demonstrated that pcDNA3.1 (+)-HBV1.3C was better than AAV/HBV plasmid in the preparation of persistent HBV transfection mouse, by HI.

\section{Acknowledgments}

We are grateful to Professor Dr. Pei-Jer Chen for providing the plasmid pAAV-HBV1.2A and Professor Dr. Dongping $\mathrm{Xu}$ for providing the plasmid pcDNA3.1(+)-HBV1.3C.

\section{Footnotes}

Authors' Contribution:This work was a collaboration among all the authors. Xiumei Li, Meijuan Chen and Yang Yang performed the experiments; Guangze Liu and Xiangping Kong provided technical guidance; Xiumei Li and Yong Xie analyzed the data; Xiumei Li and Guangze Liu wrote the paper. All authors read and approved the final version of the manuscript.

Funding/Support:This work was supported by national major science and technology project for infectious diseases of China (2012ZX10004503), science and technology program of Guangzhou (201510010004), science and technology planning project of Guangdong province (2014A020212639).

\section{References}

1. Scaglione SJ, Lok AS. Effectiveness of hepatitis B treatment in clinical practice. Gastroenterology. 2012;142(6):1360-1368 e1. doi: 10.1053/j.gastro.2012.01.044. [PubMed:22537444]

2. Liu CJ, Kao JH. Global perspective on the natural history of chronic hepatitis B: role of hepatitis B virus genotypes A to J. Semin Liver Dis. 2013;33(2):97-102. doi: 10.1055/s-0033-1345716. [PubMed: 23749665]

3. Malmstrom S, Eilard A, Larsson SB, Hannoun C, Norkrans G, Lindh M. Genotype impact on long-term virological outcome of chronic hepatitis B virus infection. J Clin Virol. 2012;54(4):321-6. doi:10.1016/j.jcv.2012.04.015. [PubMed: 22608364]

4. Cui Y, Jia J. Update on epidemiology of hepatitis B and C in China. J Gastroenterol Hepatol. 2013;28 Suppl 1:7-10. doi:10.1111/jgh.12220. [PubMed: 23855289]

5. Kosaka K, Hiraga N, Imamura M, Yoshimi S, Murakami E, Nakahara T, et al. A novel TK-NOG based humanized mouse model for the study of HBV and HCV infections. Biochem Biophys Res Commun. 2013;441(1):230-5. doi: 10.1016/j.bbrc.2013.10.040. [PubMed: 24140055]

6. Roggendorf M, Yang D, Lu M. The woodchuck: a model for thera- peutic vaccination against hepadnaviral infection. Pathol Biol (Paris). 2010;58(4):308-14. doi: 10.1016/j.patbio.2010.04.005. [PubMed: 20646874]

7. Dandri M, Lutgehetmann M. Mouse models of hepatitis B and delta virus infection. J Immunol Methods. 2014;410:39-49. doi: 10.1016/j.jim.2014.03.002. [PubMed: 24631647]

8. Dandri M, Lutgehetmann M, Petersen J. Experimental models and therapeutic approaches for HBV. Semin Immunopathol. 2013;35(1):7-21. doi: 10.1007/s00281-012-0335-7. [PubMed: 22898798]

9. Chayama K, Hayes $\mathrm{CN}$, Hiraga N, Abe H, Tsuge M, Imamura M. Animal model for study of human hepatitis viruses. J Gastroenterol Hepatol. 2011;26(1):13-8. doi: 10.1111/j.1440-1746.2010.06470.x. [PubMed: 21175788]

10. Wolff JA, Malone RW, Williams P, Chong W, Acsadi G, Jani A, et al. Direct gene transfer into mouse muscle in vivo. Science. 1990;247(4949 Pt1):1465-8. [PubMed:1690918]

11. Herweijer H, Wolff JA. Gene therapy progress and prospects: hydrodynamic gene delivery. Gene Ther. 2007;14(2):99-107. doi: 10.1038/sj.gt.3302891. [PubMed:17167496]

12. Chuai X, Wang W, Chen H, Deng Y, Wen B, Tan W. Lentiviral backbone-based hepatitis B virus replicon-mediated transfer favours the establishment of persistent hepatitis B virus infection in mice after hydrodynamic injection. Antiviral Res. 2014;101:68-74. doi:10.1016/j.antiviral.2013.10.019. [PubMed:24239872]

13. Shashidharamurthy R, Machiah D, Bozeman EN, Srivatsan S, Patel J, Cho A, et al. Hydrodynamic delivery of plasmid DNA encoding human FcgammaR-Ig dimers blocks immune-complex mediated inflammation in mice. Gene Ther. 2012;19(9):877-85. doi: 10.1038/gt.2011.175. [PubMed:22113315]

14. Suda T, Kamimura K, Kubota T, Tamura Y, Igarashi M, Kawai $\mathrm{H}$, et al. Progress toward liver-based gene therapy. Hepatol Res. 2009;39(4):325-40. doi: 10.1111/j.1872-034X.2008.00479.x. [PubMed:19207594]

15. Zhang G, Song YK, Liu D. Long-term expression of human alpha1antitrypsin gene in mouse liver achieved by intravenous administration of plasmid DNA using a hydrodynamics-based procedure. Gene Ther. 2000;7(15):1344-9. doi: 10.1038/sj.gt.3301229. [PubMed:10918507]

16. Chen ZY, Yant SR, He CY, Meuse L, Shen S, Kay MA. Linear DNAs concatemerize in vivo and result in sustained transgene expression in mouse liver. Mol Ther. 2001;3(3):403-10. doi: 10.1006/ mthe.2001.0278. [PubMed: 11273783]

17. Ketzinel-Gilad M, Zauberman A, Nussbaum O, Shoshany Y, BenMoshe O, Pappo O, et al. The use of the hydrodynamic HBV animal model to study HBV biology and anti-viral therapy. Hepatol Res. 2006;34(4):228-37. doi:10.1016/j.hepres.2006.01.008. [PubMed:16520091]

18. Yang D, Liu L, Zhu D, Peng H, Su L, Fu YX, et al. A mouse model for HBV immunotolerance and immunotherapy. Cell Mol Immunol. 2014;11(1):71-8. doi: 10.1038/cmi.2013.43. [PubMed: 24076617]

19. Tzeng HT, Tsai HF, Chyuan IT, Liao HJ, Chen CJ, Chen PJ, et al. Tumor necrosis factor-alpha induced by hepatitis B virus core mediating the immune response for hepatitis B viral clearance in mice model. PLoS One. 2014;9(7):e103008. doi: 10.1371/journal. pone.0103008. [PubMed:25047809]

20. Huang LR, Wu HL, Chen PJ, Chen DS. An immunocompetent mouse model for the tolerance of human chronic hepatitis B virus infection. Proc Natl Acad Sci U S A. 2006;103(47):17862-7. doi: 10.1073/pnas.0608578103. [PubMed:17095599]

21. Li L, Shen H, Li A, Zhang Z, Wang B, Wang J, et al. Inhibition of hepatitis $\mathrm{B}$ virus (HBV) gene expression and replication by $\mathrm{HBx}$ gene silencing in a hydrodynamic injection mouse model with a new clone of HBV genotype B. Virol J. 2013;10:214. doi: 10.1186/1743422X-10-214. [PubMed: 23805945]

22. Institute of Laboratory Animal Resources, Committee on Care, Use of Laboratory Animals, National Institutes of Health. Division of Research Resources. Guide for the care and use of laboratory animals. National Academies; 1985.

23. Sunbul M. Hepatitis B virus genotypes: global distribution and clinical importance. World J Gastroenterol. 2014;20(18):5427-34. doi:10.3748/wjg.v20.i18.5427. [PubMed:24833873] 
24. Xibing G, Xiaojuan Y, Juanhua W, Zhong H. Relationship between HBV genotypes $\mathrm{B}, \mathrm{C}$ and follicular helper T cells in patients with chronic hepatitis B and its significance. Hepat Mon 2013;13(1):e6221. doi: 10.5812/hepatmon.6221. [PubMed: 23585765]

25. Dion S, Bourgine M, Godon O, Levillayer F, Michel ML. Adeno-associated virus-mediated gene transfer leads to persistent hepatitis B virus replication in mice expressing HLA-A2 and HLA-DR1 molecules. J Virol. 2013;87(10):5554-63. doi: 10.1128/JVI.03134-12. [PubMed: 23468504]

26. Dang SS, Jia XL, Song P, Cheng YA, Zhang X, Sun MZ, et al. Inhibitory effect of emodin and Astragalus polysaccharide on the replication of HBV. World J Gastroenterol. 2009;15(45):5669-73. [PubMed: 19960563]

27. He Q, Tang Q, Le X, Lv D, Zhang X, Ao F, et al. The relationship between intrahepatic distribution of hepatitis B virus core antigen and serum ALT, HBV DNA levels and HBeAg Status. Infect Int (Electro Edit). 2012;1:84-90.
28. Chu CM, Shyu WC, Liaw YF. Immunopathology on hepatocyte expression of HBV surface, core, and $\mathrm{x}$ antigens in chronic hepatitis B: clinical and virological correlation. Dig Dis Sci. 2010;55(2):44651. doi: 10.1007/s10620-009-0895-0. [PubMed:19680810]

29. Nguyen T, Thompson AJ, Bowden S, Croagh C, Bell S, Desmond $\mathrm{PV}$, et al. Hepatitis B surface antigen levels during the natural history of chronic hepatitis B: a perspective on Asia. J Hepatol. 2010;52(4):508-13. doi: 10.1016/j.jhep.2010.01.007. [PubMed: 20206400]

30. Wang J, Wang B, Huang S, Song Z, Wu J, Zhang E, et al. Immunosuppressive drugs modulate the replication of hepatitis B virus (HBV) in a hydrodynamic injection mouse model. PLoS One 2014;9(1):e85832. doi: 10.1371/journal.pone.0085832. [PubMed: 24465734]

31. Chao DC, Hu KQ. Update on rescue therapies in patients with lamivudine-resistant chronic hepatitis B. Drug Des Devel Ther. 2013;7:777-88. doi:10.2147/DDDT.S33947. [PubMed: 23990707] 